\title{
Regional Cerebellar Volumes Predict Functional Outcome in Children with Cerebellar Malformations
}

\author{
Marie-Eve Bolduc • Adre J. du Plessis • \\ Nancy Sullivan • Nicolas Guizard • Xun Zhang • \\ Richard L. Robertson • Catherine Limperopoulos
}

Published online: 7 September 2011

(C) Springer Science+Business Media, LLC 2011

\begin{abstract}
The cerebellum has recently been recognized for its role in high-order functions, including cognition, language, and behavior. Recent studies have also begun to describe a functional topography of the mature cerebellum that includes organization on a mediolateral axis. However, no study to date has examined the relationship between
\end{abstract}

M.-E. Bolduc

School of Physical \& Occupational Therapy, McGill University, Montreal, QC, Canada

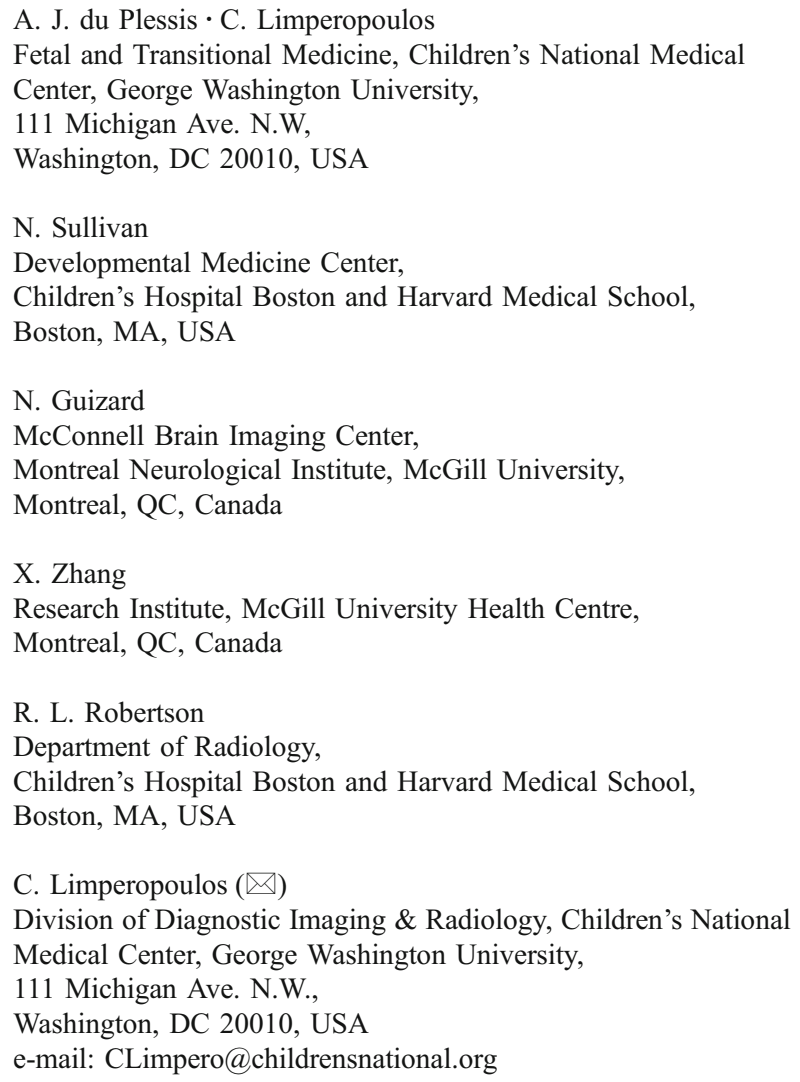

regional cerebellar volume and developmental disabilities in children with cerebellar malformations. The objective of this study was to estimate the extent to which total and regional cerebellar volumes are associated with developmental disabilities in a cohort of children with cerebellar malformations. Children aged 1 to 6 years with a diagnosis of cerebellar malformation underwent standardized outcome measures and quantitative magnetic resonance scanning. The cerebellum was parcellated into seven mediolateral zones (three for each hemisphere plus the vermis) for regional volume analysis. In children with cerebellar malformations, decreased total cerebellar volume was associated with delays in global development, expressive language, cognition, as well as gross and fine motor function. Decreased volume in the right lateral cerebellar hemisphere was related to impaired cognition, expressive language, and gross motor function. Additionally, reduced vermis volume was associated with impaired global development, cognition, expressive language, and gross and fine motor skills, as well as behavior problems and a higher rate of positive autism spectrum screening test. These results begin to define the structural topography of functional outcome in children with cerebellar malformations and should lead to greater accuracy of prognostication as well as timely early developmental interventions.

Keywords Cerebellum · Cerebellar malformations . Development Outcome $\cdot$ Magnetic resonance imaging

\section{Introduction}

It is now accepted that the cerebellum plays a role that far exceeds motor control and coordination [1,2]. Over a decade ago, Schmahmann and Sherman $[1,2]$ described the 
cerebellar cognitive affective syndrome in adults with cerebellar disorders, characterized by impairment in executive functions, visuospatial skills, language, and affect. Stimulated by these insights, subsequent studies in humans and primates have begun to define a topographic map of the different cerebellar functions [1, 3-5]. The proposed functional divisions run along both mediolateral and anterior-posterior axes. Specifically, the lateral hemispheres of the cerebellum appear to be involved in higher cognitive function. Conversely, the flocculonodular lobe and anterior vermis are primarily involved in axial motor control, and the fastigial nuclei of the posterior cerebellar lobe and posterior vermis regulate emotion, social behavior, and affect $[1,3,6,7]$.

The presence of strong anatomical connectivity between defined regions of the cerebellum and areas of the cerebrum known to be involved in these higher cognitive functions further corroborates this evidence $[8,9]$. The cerebellum's role in motor control has long been demonstrated through the presence of various anatomical pathways connecting the cerebellum to the spinal cord and the cerebral cortex in feedforward and feedback directions [10]. More recently, the use of retrograde transneuronal transport has allowed researchers to demonstrate a strong efferent association between the dentate nucleus and the prefrontal cortex through the thalamus in primates [7]. Specifically, the deep nuclei of the cerebellum are connected to the motor, supplementary motor, prefrontal, posterior parietal and superior temporal cortices, in addition to the cingulate and parahippocampal gyri through the thalamus [11]. Output connections from the dentate nucleus to the inferior parietal lobule and the dorsolateral prefrontal cortex have also been described [12]. Currently, there appears to be little or no overlap of function over the topographic organization of the mature cerebellum.

In spite of accumulating evidence of a cerebellar topographic organization, it remains unclear whether and, if so, to what extent developmental anomalies of the cerebellum affect functional abilities in young children with cerebellar malformations. In previous work, we have demonstrated that the developmental outcome of cerebellar malformations in children is variable, ranging from normal or near-normal development to severe developmental disabilities [13, 14]. However, no study to date has examined the relationship between regional cerebellar volumes and specific developmental disabilities in children with cerebellar malformations. Because the cerebellum continues to develop over the early years of life $[15,16]$, greater understanding of the potential functional deficits associated with the type and severity of the malformation could help to guide early intervention strategies with the goal of minimizing developmental disabilities and optimizing functional outcomes. The objective of this study was to estimate the extent to which total and regional cerebellar volumes are associated with global development, as well as motor, cognitive, language (expressive), and social-behavioral skills in a cohort of children with cerebellar malformations. We hypothesized that decreased total cerebellar volume is associated with global developmental delay. We also predicted that lateral cerebellar volumes are associated with cognitive, language, and motor function. Moreover, we postulated that the cerebellar vermis volume is associated with social-behavioral skills (i.e., internalizing or externalizing behavior problems and early signs of autism spectrum disorders).

\section{Patients and Methods}

\section{Procedures}

Potential candidates for this study were identified through a systematic electronic search of the MRI database at Children's Hospital Boston. We selected all English-speaking full-term children (gestational age $>37$ weeks) born between 2003 and 2008 with the prenatal or postnatal diagnosis of cerebellar malformation including: DandyWalker malformation, inferior vermis hypoplasia, cerebellar and/or vermis hypoplasia, rhombencephalosynapsis, and Joubert syndrome. The diagnostic criteria are described below:

Dandy-Walker malformation: Partial or complete absence of the cerebellar vermis with continuity between the cisterna magna and the fourth ventricle, and enlargement of the posterior fossa with upward displacement of the torcula.

Inferior vermis hypoplasia: Incomplete caudal growth of the inferior vermis over the fourth ventricle.

Cerebellar hypoplasia: Underdevelopment of one or both cerebellar hemispheres (unilateral or bilateral).

Vermis hypoplasia: A malformation that affected more than $1 / 3$ or the cerebellar vermis.

Rhombencephalosynapsis: Absent vermis and fusion of the cerebellar hemispheres.

Joubert syndrome: Presence of a molar tooth sign on axial plane, characterized by deep interpeduncular fossa, enlarged superior cerebellar peduncles that are oriented horizontally, and hypoplasia of the cerebellar vermis.

Our exclusion criteria included fetal or neonatal central nervous system infection, major intracranial birth trauma, inherited metabolic disease, or major pre- or postnatal cerebral ischemic injury.

To characterize the sample, we obtained pertinent clinical information through a systematic medical record review of 
all subjects (e.g., gender, gestational age, chromosome anomalies). Scientific and ethics approval was obtained from the Committee on Clinical Investigation at Children's Hospital Boston, and written informed consent was obtained from the child's parent or legal guardian in all cases.

\section{MRI Acquisition}

A uniform protocol for all MR scans was used, and all sequences were acquired at Children's Hospital Boston, Massachusetts, USA. Imaging was performed with a 1.5 Tesla General Electric System (GE Medical Systems, Milwaukee, WI) using a quadrature or eight-channel phased array head coil. First, through the use of an automated shimming procedure, a sagittal localizer image was acquired. Two different imaging modes were applied: a threedimensional Fourier-transform spoiled gradient recalled sequence (coronal acquisition; slice thickness, $1.5 \mathrm{~mm}$; inplane resolution, $0.78125 \times 0.78125 \mathrm{~mm}$; flip angle, $45^{\circ}$; repetition time, $35 \mathrm{~ms}$; echo time, $5 \mathrm{~ms}$; field of view, $18 \mathrm{~cm}$; matrix, $256 \times 256$; 124 slices) and a double-echo (proton density and T2-weighted) spin-echo sequence (3$\mathrm{mm}$ axial slices; repetition time, 3,000 ms; echo times, 36 and $162 \mathrm{~ms}$; field of view, $18 \mathrm{~cm}$; matrix, $256 \times 256$, interleaved acquisition; 68 slices). In situations where patients were scheduled to undergo a clinically ordered MRI scan, we performed a combined clinical research MRI study in which sedation was used which followed the institutional clinical sedation guidelines. For patients who did not have a clinical MRI scheduled, we performed a research MRI study using the same imaging parameters; however, no sedation was used, and children were studied under natural sleep.

\section{Cerebellar Diagnostic Groups}

The MRI diagnosis of cerebellar malformation and its categorization were confirmed by an experienced pediatric neuroradiologist (R.L.R.) blinded to past medical history and developmental outcome.

\section{Image Analysis}

Manual voxel labeling using Display, an in-house visualization tool developed at the McConell Brain Imaging Centre of the Montreal Neurological Institute [17], was used to outline the cerebellum. Subsequently, the cerebellum was parcellated in seven regions. Each cerebellar hemisphere was parcellated into three zones in the coronal plane, i.e., medial hemispheric, midhemispheric (lateral hemispheric zone 1 by Makris et al. [18]), lateral hemispheric (lateral hemispheric zone 2 by Makris et al.
[18]), and vermis was outlined, using a previously validated technique described by Makris and colleagues $[18,19]$. The first line was drawn by joining one point created by the intersection of the intraculminate fissure and the cerebellar margin and another point at the meeting point of the secondary fissure and the cerebellar margin. A control point was placed on the horizontal fissure at the medial one third distance between the center of the cerebellum and the hemispheric margin. The second line was drawn using the intersection of the superior posterior fissure and the cerebellar margin and a second point located at the junction of the ansoparamedian fissure and the cerebellar margin. A second control point was placed on the horizontal fissure at the lateral one third distance between the hemispheric margin and the center of the cerebellum. Three points were also positioned on each side of the vermis. The curves joining the three points were drawn using a cubic function (Fig. 1). Anatomical landmarks were identified by the same investigator (M.B.), and their position was verified by a senior investigator (C.L.) in order to reach consensus for each cerebellar landmark. Each parcellated cerebellum was then reviewed, and manual corrections were made when necessary. Volumes in cubic centimeters were calculated for the total cerebellum and each of the seven outlined regions. Examples of parcellated cerebellums in children with cerebellar malformations are presented in Fig. 2. This parcellation method was validated on the basis of anatomical landmarks, cerebellar histology and connectivity [18], and incorporated current knowledge of the functional organization of the cerebellum based on both human and primate literature [18]. Interclass coefficients were used to evaluate intra-rater and inter-rater reliability, which averaged 0.95 for both measures [19]. In addition, consistency in our volume measurements was examined by selecting five MRI scans from the current study, which were parcellated a second time using the same parcellation method, by the same investigator (M.B.). Total cerebellar volume differences averaged $0.79 \mathrm{cc}$, and volume differences in all parcellated cerebellar regions averaged $2.5 \mathrm{cc}$.

\section{Standardized Outcome Measures}

Standardized assessments were used to evaluate developmental and functional skills in all children with cerebellar malformations. These included the Mullen Scales of Early Learning [20] administered by a licensed child psychologist (N.S.), the Peabody Developmental Motor Scales [21] administered by a pediatric occupational therapist (C.L.), and the Child Behavior Checklist [22] and Modified Checklist for Autism in Toddlers [23] (caregiver reports). All evaluators were blind to MRI findings, perinatal and neonatal complications, neurological findings, and each other's clinical findings. 


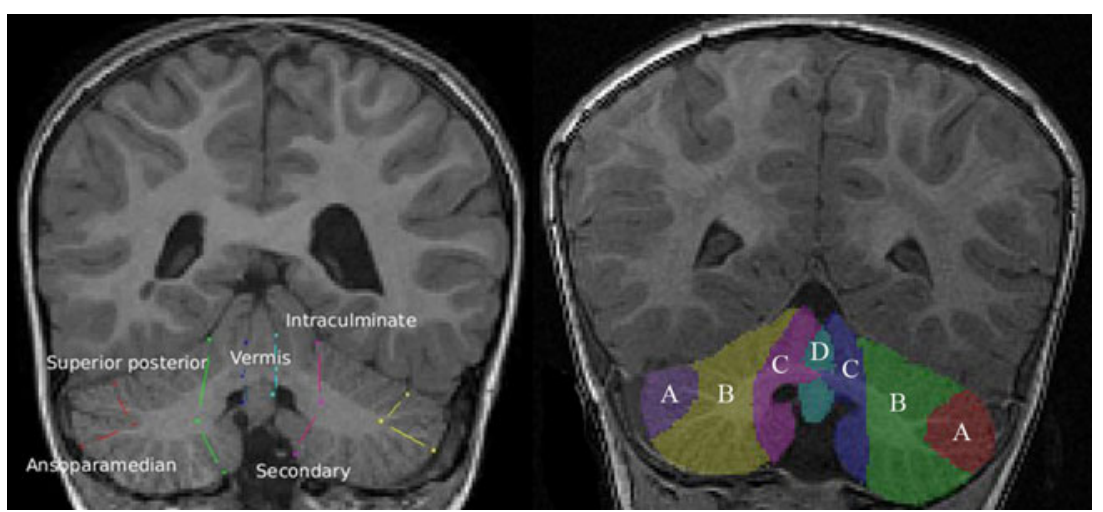

Fig. 1 An example of the parcellation scheme used to divide the cerebellum into seven regions. The image on the left is an example of the anatomical landmarks used to carry out the cerebellar parcellation. The image on the right represented the various zones: a represents the

The Mullen Scales of Early Learning [20] was used to evaluate each child's development. It is divided into five subscales including receptive language (language comprehension, auditory-spatial concepts, memory for commands, and general concepts), expressive language (spontaneous use of words or sounds and gestures), visual reception skills (visual memory and discrimination, spatial organization), and gross motor (e.g., head and trunk control, walking) and fine motor skills (unilateral and bilateral manipulation), in addition to the early learning composite quotient. In this study, we refer to visual reception skills as cognitive skills. The gross motor scores of the Mullen Scales of Early Learning are not applicable to children over 33 months. The Peabody Developmental Motor Scales [21] is a standardized test for assessing gross and fine motor function in children from birth through 83 months of age. It includes six subtests: reflexes, stationary, locomotion, object manipulation, grasping, and visual-motor integration. Continuous scores were used for analyzing the association between total and regional cerebellar volumes and functional outcomes. For all the above described assessment tools, a score right and left lateral hemispheric zones; $\mathbf{b}$ represents the right and left midhemispheric zones; $\mathbf{c}$ the right and left medial hemispheric zones; and $\mathbf{d}$ the vermis

below two standard deviations of the normative mean was defined as being indicative of an impairment when describing the developmental outcome of children with cerebellar malformations.

In addition, the Child Behavior Checklist [22] is a caregiver report that was used to assess maladaptive behaviors (e.g., anxious, depressive, aggressive, hyperactive behaviors). The total problem scale score is expressed as a $T$ score and was used as a continuous score in analyzing the association between cerebellar volume and behavioral outcomes; however, a score equal or above 60 was defined as being in the impaired range of functioning. The Modified Checklist for Autism in Toddlers [23] is a parental report used as a screening test for early detection of autistic behaviors. Failing of three items total or two critical items is used as a cutoff.

\section{Statistical Analysis}

Descriptive statistics were used to characterize our sample. Developmental, MRI, and clinical characteristics

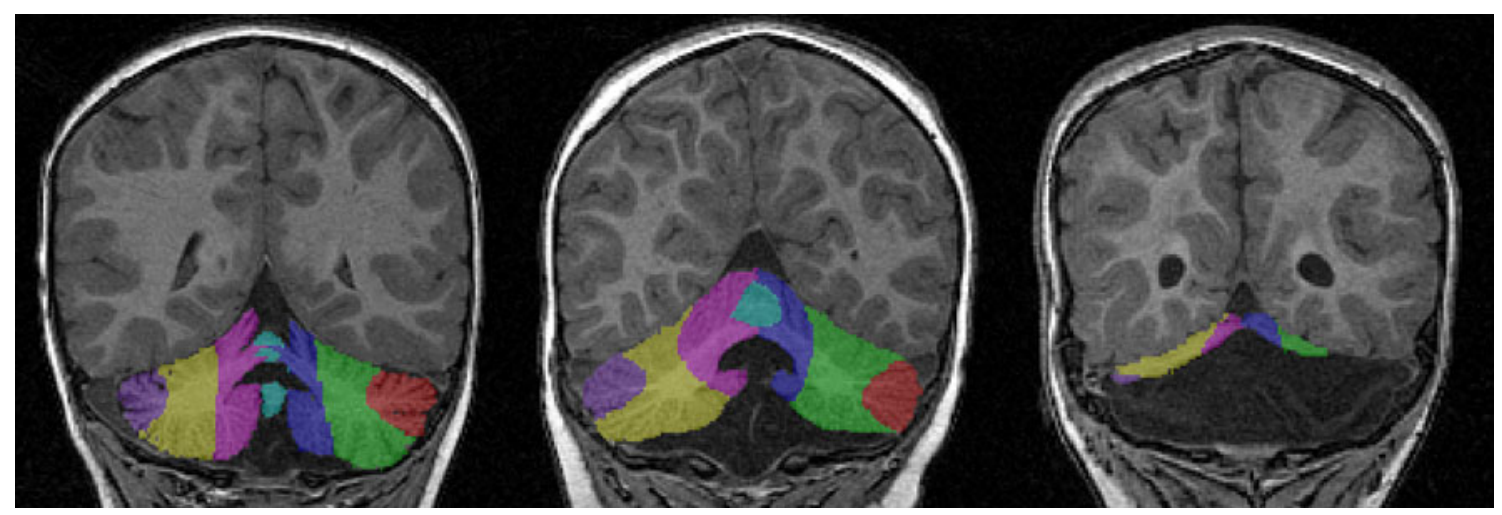

Fig. 2 Examples of cerebellar parcellations performed on coronal T1weighted spoiled gradient recalled sequences in children with varying degrees of cerebellar and/or vermis hypoplasia. On the left and center are examples of a 48-month-old child (left) and 34-month-old child (center) with vermis hypoplasia, and on the right is an example of a 54-month-old child with bilateral cerebellar and vermis hypoplasia 
of the patients were summarized using means for continuous data and proportions for categorical data. Based on a priori hypotheses, the following were selected as developmental and functional outcomes: cognitive skills (i.e., visual reception), expressive language, and early learning composite quotient of the Mullen Scales of Early Learning, gross and fine motor scores of Peabody Developmental Motor Scales, the total problem score on the Child Behavior Checklist, and total score on the Modified Checklist for Autism in Toddlers. Linear regression analyses were then used to examine the relationship between total and regional cerebellar volumes and continuous developmental and functional outcome measures (Mullen Scales of Early Learning, Peabody Developmental Motor Scales, and Child Behavior Checklist), and logistic regression analyses were used for the dichotomous outcome (Modified Checklist for Autism in Toddlers), in the overall cohort $(n=32)$, and in a subgroup of children with isolated cerebellar malformations $(n=20)$. Because of our sample size, each regional cerebellar volume was examined using a separate linear regression. Raw $p$ values are presented and associations that remain significant after Bonferroni adjustments for multiple comparisons are described because of the controversy around this correction method [24]. All assumptions for linear regressions were met. Correlations were examined to identify the presence of potential confounding variables. Known confounders were entered in all models including age, presence of associated central nervous system malformations, chromosome anomalies, and total cerebellar volume. Differences in regional volumes and outcome between children with isolated cerebellar malformations and those with cerebellar malformations and associated supratentorial/chromosome anomalies were analyzed using independent-samples $t$ test for continuous data and Pearson's chi-square for dichotomous outcome. Residual plots were examined for fits of linear regressions and the Hosmer-Lemshow test for logistic regressions. Statistical analyses were performed using SPSS Statistics version 17.0 (IBM Company, Chicago, IL, USA).

\section{Results}

Characteristics of the Cohort

Fifty-eight children with cerebellar malformations met our inclusion criteria. Of these, four died in infancy and three were lost to follow-up. Of the remaining 51 families, 48 (94\%) consented to enrollment in the study, although one consented for developmental testing only. Seven children could not complete the MRI scanning (one child died before the MRI study was performed, and six children woke up during the MRI study and the MRI study was terminated, and eight children with ventricular peritoneal shunts were excluded because their cerebellar anatomy was obscured to some extent by metal artifacts). The remaining 32 children composed the core of our study sample. However, in two subjects with severe cerebellar malformations, the anatomical landmarks for cerebellar hemispheres parcellation could not be clearly identified; therefore, in these children, we obtained only volumes for total cerebellum and vermis. The study population is presented in Fig. 3.

The clinical radiological diagnoses of our sample are summarized in Table 1. The mean (standard deviation) gestational age was 39.2 weeks (2.2), and the mean birth weight was $3,426.6 \mathrm{~g}$ (674.8). Children underwent developmental testing at a mean age of 28.8 months (14.8; median, 24.0 months; range, 12-73 months) and MRI scans at a mean of 28.0 months (16.7; median, 22.0 months; range, 10-72 months). The mean (standard deviation) age difference between developmental testing and MRI scans was 5.3 months (3.5). Our sample was composed of 19 males (59\%) and 13 females (41\%). Twenty children $(63 \%)$ had isolated cerebellar malformations, and 12 children (37\%) had associated supratentorial abnormalities and/or chromosomal findings (24\%) including agenesis/hypoplasia of the corpus callosum (7), abnormal gyral pattern (3), nodular heterotopias (2), decreased white matter (1), absence of septum pellucidum (1), periventricular white matter abnormalities (1), and ventriculomegaly (1). Chromosomal abnormalities included Joubert syndrome (1), CHARGE syndrome (2), and chromosome 10 anomalies (1). Developmental testing for children with isolated cerebellar malformation was performed at the mean age of 29.1 months (14.8; median, 26 months; range, 12-63 months) and MRI scans at a

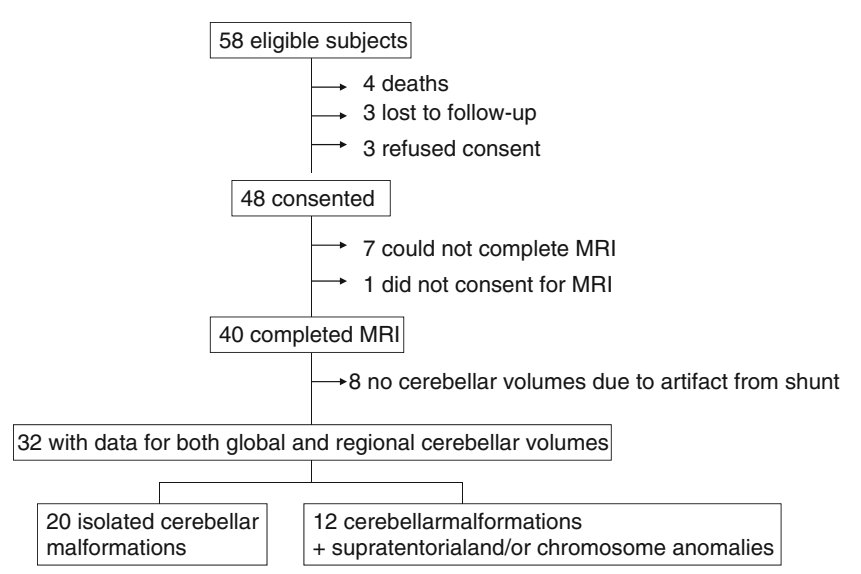

Fig. 3 Flow chart detailing the study population 
Table 1 Cerebellar malformation diagnostic groups

\begin{tabular}{ll}
\hline Diagnostic group & $\begin{array}{l}\text { Frequency (\%) } \\
N=32\end{array}$ \\
\hline Cerebellar hypoplasia & $11(34 \%)$ \\
Inferior vermis hypoplasia & $11(34 \%)$ \\
Vermis hypoplasia & $5(16 \%)$ \\
Rhombencephalosynapsis & $3(9 \%)$ \\
Dandy-Walker malformation & $1(3 \%)$ \\
Joubert syndrome & $1(3 \%)$ \\
\hline
\end{tabular}

mean age of 29.1 months (16.4; median, 29 months; range, 10-62 months).

\section{Developmental Outcomes of the Cohort}

Two thirds (17/27) of children with cerebellar malformations experienced important gross motor disabilities, and 16 children (50\%) experienced delayed global development and cognitive impairments. Additionally, almost half the cohort (15/32 children) demonstrated delays in expressive language skills and 13 children (41\%) in receptive language skills. Fine motor function was impaired in 12 children, and behavioral problems (Child Behavior Checklist) were present in just under half (15) of the children. Finally, 19 children $(59 \%)$ had a positive screening for early signs of autism features (Modified Checklist for Autism in Toddlers). A more detailed description of the functional outcome of the children included in this study has been described previously [9]. Given the proportion of children with associated supratentorial anomalies and chromosomal abnormalities in our cohort $(n=12)$, we also compared the developmental outcomes of those with isolated cerebellar malformations $(n=20)$ and those with combined supratentorial/chromosomal abnormalities. Overall, children with cerebellar malformations and associated supratentorial anomalies and chromosomal abnormalities presented significantly greater developmental and functional impairments and disabilities when compared to those with isolated cerebellar malformations on all outcome measures except on the total problem scale of the Child Behavior Checklist (Table 2).

Total and Regional Cerebellar Volumes in Children with Cerebellar Malformations

The total and regional cerebellar volume measures of our sample are presented in Table 3. Total and regional volumes in children with isolated cerebellar malformations were compared to those children with associated supratentorial/ chromosome anomalies. The results are presented in Table 4.
Association Between Total and Regional Cerebellar Volumes and Outcome in the Overall Cohort

We first examined the relationship between total and regional cerebellar volumes and developmental outcomes in our entire cohort, controlling for the presence of supratentorial and/or chromosomal anomalies and age at testing for all analyses. We also controlled for total cerebellar volume when analyzing the effect of regional cerebellar volumes on developmental outcomes. Decreased total cerebellar volume was associated with deficits in gross motor function. Volumetric loss in the right lateral hemispheric zone was associated with expressive language and gross motor deficits. It is noteworthy that decreased right lateral volume was a stronger predictor of outcome than the presence of supratentorial/chromosome anomalies for all developmental/functional domains. Additionally, reduced vermis volume was associated with global developmental delays, as well as deficits in cognition, language, gross motor skills, and behavioral problems. Finally, reduced vermis volumes were associated with a higher rate of autistic features. Importantly, vermis volume was a more important predictor of functional outcome than the presence of supratentorial/chromosome anomalies on all functional outcomes except the autism spectrum disorder screening. There were no statistically significant associations between medial hemispheric and midhemispheric zones and functional outcome. After correcting for multiple analyses using the Bonferroni method [25], smaller vermis volume remained predictive of global developmental delays, as well as cognition, language, gross motor, and behavioral problems. The relationship between total and regional cerebellar volumes and developmental outcomes is detailed in Table 5.

Association Between Total and Regional Cerebellar

Volumes and Outcome in Children with Isolated

Cerebellar Malformations

\section{Total Cerebellar Volume and Outcome}

We then examined the relationship between total and regional cerebellar volume and global development and functional skills, controlling for age, in children with isolated cerebellar malformations (i.e., no associated supratentorial or chromosomal abnormalities). On bivariate analyses, decreased total cerebellar volumes were associated with global developmental delay $(p=0.002)$ and cognitive delays $(p=0.01)$ and with deficits in gross and fine motor skills ( $p=0.03$ for both), as well as with expressive language skills $(p=0.01)$. The association between total cerebellar volume and global development remained significant when adjusting for multiple comparisons. 
Table 2 Comparison of outcomes in children with isolated cerebellar malformation versus those with CNS or chromosomal anomalies

${ }^{a}$ Indicates a statistically significant difference in outcomes between children with isolated cerebellar malformation versus those with CNS or chromosomal anomalies
Isolated cerebellar

malformation

Mean (SD) $N=20$
Cerebellar malformation with associated $p$ value CNS or chromosome anomaly

Mean (SD) $N=12$

\begin{tabular}{|c|c|c|c|}
\hline $\begin{array}{l}\text { Age at developmental testing } \\
\text { (months) }\end{array}$ & $28.8(14.8)$ & $29.1(14.8)$ & \\
\hline \multicolumn{4}{|c|}{ Peabody Developmental Motor Scales } \\
\hline Gross motor & $76.00(9.87)$ & $65.33(1.16)$ & $<0.001$ \\
\hline Fine motor & $78.95(7.78)$ & $68.33(4.98)$ & $<0.001$ \\
\hline \multicolumn{4}{|l|}{ Mullen Scales of Early Learning } \\
\hline Cognitive skills & $41.70(5.18)$ & $24.08(7.87)$ & 0.001 \\
\hline Expressive language & $36.80(12.15)$ & $26.17(10.30)$ & $0.02^{\mathrm{a}}$ \\
\hline Early learning composite & $83.00(20.43)$ & $59.17(15.87)$ & 0.002 \\
\hline \multicolumn{4}{|l|}{ Child Behavior Checklist } \\
\hline Total problem scale & $49.50(11.77)$ & $57.67(10.89)$ & 0.06 \\
\hline \multicolumn{4}{|l|}{$\begin{array}{l}\text { Modified Checklist for Autism } \\
\text { in Toddlers }\end{array}$} \\
\hline Total score & $3.95(4.71)$ & $9.08(5.27)$ & 0.004 \\
\hline
\end{tabular}

\section{Lateral Hemispheric Volume and Outcome}

When examining laterality (left versus right lateral hemispheric volume), decreased volume in the right lateral hemisphere was associated with deficits in cognition, expressive language, and gross motor skills. Conversely, there was no association between reduced cerebellar volume in the left lateral hemisphere and specific functional skills in children with isolated cerebellar malformations. These associations did not remain significant following Bonferroni correction. The results are described in Table 6 .

\section{Midhemispheric Volume and Outcome}

There were no significant associations between midhemispheric volumes and developmental and functional scores in children with isolated cerebellar malformations.

Table 3 Total and regional cerebellar volumetric measurements of our cohort $(N=32)$

\begin{tabular}{lcc}
\hline $\begin{array}{l}\text { Total and regional cerebellar } \\
\text { volumes (cc) }\end{array}$ & Mean (SD) (cc) & Range (cc) \\
\hline Total cerebellar volume & $83.88(42.63)$ & $3.22-166.67$ \\
Right lateral hemispheric & $5.92(3.19)$ & $0.42-12.35$ \\
Left lateral hemispheric & $6.40(3.06)$ & $0.60-13.76$ \\
Right midhemispheric & $21.87(10.15)$ & $2.61-44.70$ \\
Left midhemispheric & $23.44(10.10)$ & $2.46-49.65$ \\
Right medial hemispheric & $14.19(7.44)$ & $1.09-26.45$ \\
Left medial hemispheric & $15.00(7.08)$ & $1.87-30.72$ \\
Vermis & $3.20(2.36)$ & $0.00-9.15$ \\
\hline
\end{tabular}

$S D$ standard deviation

\section{Medial Hemispheric Volume and Outcome}

There were no significant associations between medial hemispheric volumes and developmental and functional outcome in our cohort of children with isolated cerebellar malformations.

\section{Vermis Volume and Outcome}

There was a highly significant relationship between decreased vermis volume and global developmental delay, cognition, expressive language, gross motor, and fine motor. Moreover, smaller vermis volumes were associated with behavioral problems and a higher prevalence of early signs of autistic features on the Modified Checklist for Autism in Toddlers. The associations between vermis volume and global development, cognition, expressive language, and gross and fine motor skills remained significant after Bonferroni corrections. The relationship between vermis volume and the outcome measures is summarized in Table 7.

\section{Discussion}

Currently used diagnostic categories for cerebellar malformations are often associated with a wide range of neurodevelopmental outcomes which frequently limit reliable prognostication $[13,26]$. To our knowledge, this is the first report in which advanced quantitative cerebellar MRI volumetric and parcellation techniques have been used to examine the relationship between regional cerebellar volumes and developmental and functional outcomes. Our 
Table 4 Comparison of cerebellar volumes in children with isolated cerebellar malformation versus those with CNS or chromosomal anomalies

${ }^{a}$ Indicates a statistically significant volume difference between children with isolated cerebellar malformation versus those with CNS or chromosomal anomalies

\begin{tabular}{lcll}
$\begin{array}{l}\text { Total and regional cerebellar } \\
\text { volumes }(\mathrm{cc})\end{array}$ & $\begin{array}{l}\text { Isolated cerebellar } \\
\text { malformation } \\
\text { Mean (SD) } N=20\end{array}$ & $\begin{array}{l}\text { Cerebellar malformations with associated } \\
\text { CNS or chromosome anomaly } \\
\text { Mean (SD) } N=12\end{array}$ & $p$ value \\
\hline Total cerebellar volume & $96.66(38.92)$ & $62.58(41.39)$ & $0.03^{\mathrm{a}}$ \\
Right lateral hemispheric & $6.57(3.40)$ & $4.64(2.37)$ & 0.12 \\
Left lateral hemispheric & $6.86(3.14)$ & $5.38(2.75)$ & 0.23 \\
Right midhemispheric & $23.09(1.03)$ & $19.41(9.88)$ & 0.36 \\
Left midhemispheric & $24.42(9.58)$ & $21.26(11.44)$ & 0.48 \\
Right medial hemispheric & $15.36(7.93)$ & $11.86(6.04)$ & 0.23 \\
Left medial hemispheric & $16.44(7.24)$ & $11.80(5.87)$ & 0.10 \\
Vermis & $4.09(2.33)$ & $1.70(1.55)$ & $0.004^{\mathrm{a}}$
\end{tabular}

study provides new insights into the functional topography of disturbed cerebellar growth in the developing child. Specifically, decreased total cerebellar volume was associated with global developmental delay, cognitive and language impairments, motor deficits, and functional disabilities in children with isolated cerebellar malformations. Our data also demonstrate important relationships between regional cerebellar volumes and domain-specific developmental and functional skills. The most striking finding is the apparent pervasive functional impact of disturbed cerebellar vermis growth at this young age. The wideranging impact of decreased vermis volume in both motor and non-motor functions, including deficits in global development, cognition, expressive language, and behav- ioral skills, is remarkable. Additionally, decreased vermis volume was associated with a higher prevalence of early signs of autistic features. We also showed that the lateral zones of the cerebellum, specifically the right lateral hemisphere, are associated with specific and lateralized dysfunction. Expressive language, cognition, and gross motor function were significantly affected by reduced volume of the lateralmost region of the right but not left cerebellar hemisphere. Conversely, there was no significant association between cerebellar volumes in the medial hemispheric and midhemispheric regions and specific functional skills.

Recent evidence from primates, adults, and older children links the vermis to motor, cognitive, and behav-

Table 5 Statistically significant associations between total and regional cerebellar volumes and developmental outcomes in children with cerebellar malformations $(N=32)$

\begin{tabular}{|c|c|c|c|c|}
\hline Regional cerebellar volumes (cc) & Developmental outcomes & Estimate of effect ${ }^{\mathrm{a}}$ & 95\% Confidence interval & $p$ value \\
\hline \multirow[t]{2}{*}{ Total cerebellum } & \multicolumn{4}{|c|}{ Peabody Developmental Motor Scales } \\
\hline & Gross motor function & 0.09 & $0.01-0.16$ & 0.02 \\
\hline \multirow[t]{4}{*}{ Right lateral hemispheric zone } & \multicolumn{4}{|c|}{ Mullen Scales of Early Learning } \\
\hline & Expressive language & 3.18 & $0.87-5.50$ & 0.01 \\
\hline & \multicolumn{4}{|c|}{ Peabody Developmental Motor Scales } \\
\hline & Gross motor function & 1.92 & $0.24-3.61$ & 0.03 \\
\hline \multirow[t]{10}{*}{ Vermis } & \multicolumn{4}{|c|}{ Mullen Scales of Early Learning } \\
\hline & Early learning composite & 5.06 & $2.14-7.97$ & 0.001 \\
\hline & Cognitive skills & 4.17 & $2.38-5.96$ & $<0.001$ \\
\hline & Expressive language & 3.11 & $1.25-4.97$ & 0.002 \\
\hline & \multicolumn{4}{|c|}{ Peabody Developmental Motor Scales } \\
\hline & Gross motor function & 2.77 & $1.64-3.90$ & $<0.001$ \\
\hline & \multicolumn{4}{|l|}{ Child Behavior Checklist } \\
\hline & Total problem scale & -3.55 & $(-5.25)-(-1.85)$ & $<0.001$ \\
\hline & \multicolumn{4}{|c|}{$\begin{array}{l}\text { Modified Checklist for Autism } \\
\text { in Toddlers }\end{array}$} \\
\hline & Total score & $0.53^{\mathrm{b}}$ & $0.30-0.93$ & 0.03 \\
\hline
\end{tabular}

${ }^{\text {a }}$ Estimate of effect: relationship between total and regional cerebellar volumes and functional outcome

${ }^{\mathrm{b}}$ Odds ratio and confidence interval for the odds ration from logistic regression 
Table 6 Statistically significant associations between right lateral cerebellar volume and developmental outcomes in children with isolated cerebellar malformations $(N=20)$

\begin{tabular}{|c|c|c|c|}
\hline Outcome measures & $\begin{array}{l}\text { Estimate of } \\
\text { effect }^{\mathrm{a}}\end{array}$ & $\begin{array}{l}95 \% \text { Confidence } \\
\text { interval }\end{array}$ & $p$ value \\
\hline \multicolumn{4}{|l|}{$\begin{array}{l}\text { Mullen Scales of Early } \\
\text { Learning }\end{array}$} \\
\hline Cognitive skills & 2.96 & $0.14-5.78$ & 0.04 \\
\hline Expressive language & 23.00 & $0.77-5.22$ & 0.01 \\
\hline \multicolumn{4}{|c|}{$\begin{array}{l}\text { Peabody Developmental } \\
\text { Motor Scales }\end{array}$} \\
\hline Gross motor & 2.35 & $0.25-4.44$ & 0.03 \\
\hline
\end{tabular}

${ }^{a}$ Estimate of effect: relationship between right lateral cerebellar volume and developmental outcome

ioral functions. The vermis and the fastigial nuclei have been implicated in the regulation of emotions, affect, and behavior in adults $[1,27,28]$. Two previous studies have reported a significant association between severe malformations of the cerebellar vermis and intelligence quotient $[29,30]$; however, these studies used a qualitative description of the vermis malformation. Moreover, tumor resection in the region of the cerebellar vermis is associated with significant difficulties in behavior and attention $[1,31,32]$ as well as mutism and dysarthria [1,32]. There is also evidence that the midline structures are involved in motor function, gait, and posture [4, 33]. A recent study of intrinsic connectivity networks also demonstrated the presence of vermian contribution in salience detection and sensorimotor function [8]. Finally, there is also a growing body of data demonstrating a link between the vermis and autism spectrum disorders [34-38]. In a previous study, our group demonstrated an association between cerebellar vermis injury and a positive autism screening in survivors of extreme preterm birth [39]. Nonetheless, controversy remains regarding the contribution of the cerebellar vermis in autism spectrum disorders and whether hypoplasia vs. hyperplasia of the cerebellar vermis play different roles in the development of autism spectrum disorders [40, 41]. This current study adds to the evidence that there is a significant relationship between the vermis and a positive screening for early signs of autistic features in children with cerebellar malformations. However, it is important to note that this association did not remain significant when adjusting for multiple comparisons. The results of our current study also support the important association between the cerebellar vermis region and global development, cognitive, language, and motor function, as well as behavior outcomes. Striking in our study was the strong association between reduced vermis volume and a broad spectrum of cognitive, behavioral, language, and motor dysfunction. This compelling finding suggests that during early development, structural elements in the midline cerebellum play an important role in the subsequent development of a broad spectrum of neurologic functions. In the mature brain, these functions have a more distributed topography, with less concentration in the midline region of the cerebellum. The importance of the different vermis lobules in the development of various motor and cognitive disabilities in addition to autism spectrum disorders warrants further studies.

In contrast to our current findings, children in our earlier studies with inferior vermis hypoplasia had a largely favorable outcome [42]. The reason for these differences is unclear but could potentially be related to the quantitative versus qualitative methodological differences between the studies, or more fundamental differences (e.g., isolated hypoplasia versus dysgenesis) between lesions in these studies. This is an important question that is in need of more detailed study. It is also important to note that inferior vermis hypoplasia has been considered a normal variant by some authors, although a recent study described mild functional disabilities in a subset of children [42].
Table 7 Statistically significant associations between cerebellar vermis volume and developmental outcomes in children with isolated cerebellar malformations $(N=20)$

${ }^{a}$ Estimate of effect: relationship between vermis volume and developmental outcome

${ }^{\mathrm{b}}$ Odds ratio and confidence interval for the odds ratio from logistic regression

\begin{tabular}{lccc}
\hline Outcome measures & Estimate of effect & \\
\hline Mullen Scales of Early Learning & & $95 \%$ Confidence interval & $p$ value \\
Early learning composite & 5.00 & & 0.004 \\
Cognitive skills & 4.78 & $1.85-8.14$ & $<0.001$ \\
Expressive language & 3.03 & $0.081-5.25$ & 0.01 \\
Peabody Developmental Motor Scales & 3.40 & $1.71-5.03$ & 0.001 \\
Gross motor & 2.01 & $0.49-3.54$ & 0.01 \\
Fine motor & & & 0.01 \\
Child Behavior Checklist & -3.047 & $(-5.20)-(-0.90)$ & \\
Total problem scale & & & $0.21-0.97$ \\
M-CHAT & $0.45^{\mathrm{b}}$ & & \\
Total score &
\end{tabular}


In previous literature, the medial zones have been shown to be associated with maintenance of balance, sensorimotor function, cognition, and eye movement [18, 31, 43, 44]. In our cohort, we did not find a significant relationship between the medial hemispheric zone and our developmental outcomes. One potential explanation for this may be the young age at which our children were tested (mean age, 29 months), at a time when domain-specific development and skill differentiation are still actively underway. Longterm follow-up of our cohort will assist in elucidating the role of the medial cerebellum in child development.

Available evidence from adults and older children suggests that the lateral hemispheres are associated with cognitive tasks, language, and motor control of the upper limbs [4, 10, 18, 31, 45-47]. Functional neuroimaging studies in adults show that several specific lobules located in the right lateral zones are involved in language [33]. Additionally, two previous studies in older children with tumor resection of the lateral cerebellar hemisphere(s) have reported that the right hemisphere is involved in auditory sequential memory and language, whereas the left hemisphere is linked to visual and spatial sequential memory $[31,32]$. However, it is important to note that these studies did not measure regional cerebellar volumes. Moreover, the midhemispheric zone has not been studied independently from the lateral hemispheric zone in children or adults with cerebellar lesions or malformations. Our findings support the role of the lateral cerebellar hemispheres in cognitive skills and language and also corroborate the stronger association between language skills and the right cerebellar hemisphere [31, 32]. In our study, the absence of cognitive impairment associated with decreased volume in the midhemispheric zone could be explained by the young age of the children, in whom several cognitive skills are still emerging. Alternatively, cerebellar plasticity in this group of young children with developmental lesions could result in functional reorganization of the cerebellum.

This is the first study to show an association between volumetric loss, measured by advanced three-dimensional quantitative MRI in the cerebellum, and specific functional deficits using standardized outcome measures. However, our study also has several limitations. First, although this study represents one of the largest samples of children with cerebellar malformations, it may have lacked the statistical power to detect more specific regional volume-function relationships. Second, given the young age of our cohort, many critical language and cognitive functions are not completely developed or may not be differentiated at the current developmental level, thus precluding more detailed structure-function analyses. It is also important to note that we did not specifically assess the relative contribution of occulomotor deficits on regional cerebellar volumetric loss and functional performance. This important question awaits further study. The difference in age at developmental testing and at MRI scan may have affected our results. Furthermore, in the subgroup of children with isolated cerebellar malformations in our cohort, although based on the conventional MRI studies that were reviewed by an experienced pediatric neuroradiologist there was no evidence of associated supratentorial parenchymal injury/ lesions, it is possible that subtle supratentorial injury (below the resolution of current MRI) went undetected, and that this may have confounded our structure-functional correlations. Additionally, given the cross-sectional nature of the study design, long-term follow-up of these children will be necessary in order to address this important question. Although the young age at which the children were tested may have precluded the evaluation of more detailed structure-function associations, examining the relationship between regional cerebellar volumes and specific developmental and functional skills in this age group is essential in order to implement more targeted early intervention programs. Follow-up testing at school age using higherlevel cognitive standardized outcome measurements will provide a greater appreciation of the specific skills that are impaired (e.g., perception vs. cognition). The evolution of these cerebellar structural-functional impairments and the extent to which they are transient or persistent over time may be addressed as part of these longitudinal studies. Although the results of this study successfully demonstrate the topographical organization of the cerebellum in young children with cerebellar malformations, ongoing work is needed using a larger sample size to examine the relative contribution of discrete cerebellar regions (e.g., anterior/ posterior vermis, anterior/posterior lobe) and specific functional skills. Moreover, the use of a validated cerebellar parcellation scheme that incorporates both the phylogenetical segmentation of the cerebellum and its intrinsic connectivity networks may further expand our understanding of cerebellar structure-function correlates. Lastly, future studies examining the relationship between the cerebellum and the cerebrum using tractography will also offer important insights into the role of impaired cerebellocerebro connectivity and functional disabilities in children with cerebellar malformations.

\section{Conclusion}

In summary, this study demonstrates that the mediolateral topographical organization of the cerebellum (previously described in adults) has important similarities in young children with cerebellar malformations. Specifically, we show a significant relationship between regional volumetric growth and global and domainspecific developmental and functional deficits in our 
cohort. Most remarkable is the broad functional spectrum of functional deficits, including modulation of both motor and non-motor functions, seen in children with reduced vermian volume. These data suggest that a priori knowledge based on the location and extent of regional cerebellar volumetric loss may increase the prognostic accuracy in infant with cerebellar malformations. Secondly, given that the cerebellum has a protracted development over the early years of life, a greater understanding of the potential functional deficits associated with the type and severity of the malformation may assist in guiding targeted early intervention strategies (prior to the completion of cerebellar development), using individualized rehabilitation programs that anticipate and capture the spectrum of developmental disabilities experienced by children with cerebellar malformations, so as to minimize long-term morbidity, optimize function, and maximize quality of life. Likewise, improved knowledge of the needs of children with cerebellar malformations will facilitate proper resource allocation.

In summary, our compelling structure-function data provide the first scientific evidence of the topographical organization of the cerebellum in children with cerebellar malformations. These critical, previously unavailable data can be used by health professionals to assist in developing specific early intervention therapies based on prior knowledge of the topography of the malformation, taking full advantage of the protracted development of the cerebellum and its high plasticity, treatment plans may be implemented earlier and individually tailored to target specific developmental domains in order to optimize function in children with cerebellar malformations.

Acknowledgments This study was supported by the Lifebridge Fund, the Caroline Levine Foundation, and the Trust Family Foundation. Marie-Eve Bolduc received support from a McGill University Health Centre Research Institute Scholarship. Catherine Limperopoulos [Canada Research Chair in Brain and Development (Tier II)] is supported by the Canada Research Chairs Program. Dr. du Plessis is supported through National Institutes of Health grant 1K24NS057568-01. We thank Dr. Nicol Korner-Bitensky for her methodological expertise while designing the study and for reviewing the manuscript. We also thank Shaye Moore for assistance with manuscript preparation. We are very grateful to the children and their families for participating in this study.

Conflict of Interest Statement The authors of this manuscript do not have any conflict of interest to disclose.

\section{References}

1. Schmahmann JD. Disorders of the cerebellum: ataxia, dysmetria of thought, and the cerebellar cognitive affective syndrome. J Neuropsychiatry Clin Neurosci. 2004;16:367-78.
2. Schmahmann JD, Sherman JC. The cerebellar cognitive affective syndrome. Brain Dev. 1998;121:561-79.

3. Desmond JE, Gabrieli JDE, Glover GH. Dissociation of frontal and cerebellar activity in a cognitive task: evidence for a distinction between selection and search. NeuroImage. 1998;7(4):368-76.

4. Konczak J, Timman D. The effect of damage to the cerebelum on sensorimotor and cognitive function in children and adolescents. Neuroscience Behav Rev. 2007;31:1101-13.

5. Schmahmann JD. From movement to thought: anatomic substrates of the cerebellar contribution to cognitive processing. Hum Brain Mapp. 1998;4:174-98.

6. Joyal CC, Pennanen C, Tiihonen E, Laakso MP, Tiihonen J. MRI volumetry of the vermis and the cerebellar hemispheres in men with schizophrenia. Psychiatry Res: Neuroimaging. 2004;131:11524.

7. Middleton FA, Strick PL. Cerebellar projections to the prefrontal cortex of the primate. J Neurosci. 2001;21(2):700-12.

8. Habas C, Kamdar N, Nguyen D, Prater K, Beckmann CF, Menon $\mathrm{V}$, et al. Distinct cerebellar contributions to intrinsic connectivity networks. J Neurosci. 2009;29(26):8586-94.

9. Ramnani N, Miall C. Expanding cerebellar horizons. Trends Cogn Sci. 2001;5(4):135-6.

10. Leiner HC, Leiner AL. Reappraising the cerebellum: what does the hindbrain contributes to the forebrain. Behav Neurosci. 1989;103(5):998-1008.

11. Schmahmann JD, Pandya DN. The cerebrocerebellar system. Int Rev Neurobiol. 1997;41:31-60.

12. Allen G, McColl R, Barnard H, Ringe WK, Fleckenstein J, Cullum CM. Magnetic resonance imaging of the cerebellarprefrontal and cerebellar-parietal functional connectivity. NeuroImage. 2005;28:39-48.

13. Bolduc ME, Limperopoulos C. Neurodevelopmental outcomes in children with cerebellar malformations: a systematic review. Dev Med Child Neurol. 2009;51(4):256-67.

14. Bolduc ME, Du Plessis AJ, Sullivan N, Khwaja OS, Zhang X, Barnes $\mathrm{K}$, et al. Spectrum of neurodisabilities in children with cerebellar malformations. Dev Med Child Neurol. 2011;53(5):409-16.

15. Barkovich AJ. Pediatric neuroimaging. 4th ed. Philadelphia: Lippincott Williams \& Wilkins; 2005.

16. Limperopoulos C, du Plessis AJ. Disorders of cerebellar growth and development. Curr Opin Pediatr. 2006;18(6):621-7.

17. MacDonald D. MNI-Display: Program for Display and Segmentation of Surfaces and Volumes. [Technical report] 2003 [updated January 20]; Available from: www.bic.mni.mcgill.ca/ stever/ Software/RelNotes/Display.ps

18. Makris N, Hodge SM, Haselgorve C, Kennedy DN, Dale A, Fischl B, et al. Human cerebellum: surface-assisted cortical parcellation and volumetry with magnetic resonance imaging. $\mathrm{J}$ Cogn Neurosci. 2003;15(4):584-99.

19. Makris N, Schelerf JE, Hodge SM, Haselgorve C, Albaugh MD, Seidman LJ, et al. MRI-based surface-assisted parcellation of human cerebellar cortex: an anatomically specified method with estimate of reliability. NeuroImage. 2005;25:1146-60.

20. Mullen EM. Mullen scales of early learning (AGS edn.). Circle Pines: American Guidance Service; 1995.

21. Folio RM, Fewell RR. Peabody Developmental Motor Scales and Activity Cards. Austin: DLM Teaching Resources; 1983.

22. Achenbach TM, Rescorla L. Manual for the Child Behavior Checklist. Burlington: VT University of Vermont Department of Psychiatry; 2000.

23. Robins DL, Fein D, Barton ML. The Modified-Checklist for Autism in Toddlers. Self-published 1999.

24. Perneger TV. What's wrong with Bonferroni adjustments. Br Med J. 1998;316:1236-8.

25. Bland JM, Altman DG. Multiple significance tests: the Bonferroni method. Br Med J. 1995;310:170. 
26. Tavano A, Grasso R, Gagliardi C, Triulzi F, Bresolin N, Fabbro F, et al. Disorders of cognitive and affective development in cerebellar malformations. Brain. 2007;130:2646-60.

27. Schutter DJ, van Honk J. The cerebellum on the rise in human emotion. Cerebellum. 2005;4(4):290-4.

28. Hu D, Shen H, Zhou Z. Functional asymmetry in the cerebellum: a brief review. Cerebellum. 2008;7:304-13.

29. Boddaert N, Klein O, Ferguson N, Sonigo P, Parisot D, HertzPannier L, et al. Intellectual prognosis of the Dandy-Walker malformation in children: the importance of vermian lobulation. Neuroradiology. 2003;45(5):320-4.

30. Klein O, Pierre-Kahn A, Boddaert N, Parisot D, Brunelle F Dandy-Walker malformation: prenatal diagnosis and prognosis. Childs Nerv Syst. 2003;19:484-9.

31. Levisohn L, Cronin-Golomb A, Schmahmann JD. Neuropsychological consequences of cerebellar tumor resection in children: cerebellar cognitive affective syndrome in a pediatric population. Brain. 2000;123:1041-50.

32. Riva D, Giorgi C. The cerebellum contributes to higher functions during development: evidence from a series of children surgically treated for posterior fossa tumors. Brain. 2000;123:1051-61.

33. Stoodley CJ, Schmahmann JD. Functional topography in the human cerebellum: a meta-analysis of neuroimaging studies. NeuroImage. 2009;44:489-501.

34. Courchesne E, Townsend J, Saitoh O. The brain in infantile autism. Neurology. 1994;44:214.

35. Courchesne E, Yeung-Courchesne R, Press GA, Hesselink JR, Jernigan TL. Hypoplasia of cerebellar vermal lobules VI and VII in autism. N Engl J Med. 1988;318:1349-54.

36. Filipek PA. Quantitative magnetic resonance in autism: the cerebellar vermis. Curr Opin Neurol. 1995;8:134-8.

37. Piven J, Saliba K, Bailey J, Arndt S. An MRI study of autism: the cerebellum revisited. Neurology. 1997;49:546-51.
38. Webb SJ, Sparks BF, Friedman S, Shaw DW, Giedd J, Dawson G, et al. Cerebellar vermal volumes and behavioral correlates in children with autism spectrum disorder. Psychiatry Res. 2009;172 (1):61-7.

39. Limperopoulos C, Bassan H, Gauvreau K, Robertson RLJ, Sullivan NR, Benson CB, et al. Does cerebellar injury in premature infants contribute to the high prevalence of long-term cognitive, learning, and behavioral disability in survivors? Pediatrics. 2007;120(3):584-93.

40. Hallahan B, Daly EM, McAlonan G, Loth E, Toal F, O'Brien F, et al. Brain morphometry volume in autistic spectrum disorder: a magnetic resonance imaging study of adults. Psychol Med. 2008;8:1-10.

41. Amaral DG, Schumann CM, Nordahl CW. Neuroanatomy of autism. Trends Neurosci. 2008;31(3):137-45.

42. Limperopoulos C, Robertson RL, Estroff JA, Barnewolt C, Levine $\mathrm{D}$, Bassan H, et al. Diagnosis of inferior vermian hypoplasia by fetal magnetic resonance imaging: potential pitfalls and neurodevelopmental outcome. Am J Obstet Gynecol. 2006;194 (4):1070-6.

43. Schmahmann JD, Doyon J, Toga A, Petrides M, Evans A. MRI atlas of the human cerebellum. San Diego: Academic; 2000.

44. Schmahmann JD. Dysmetria of thought: clinical consequences of cerebellar dysfunction on cognition and affect. Trends Cogn Sci. 1998;2(9):362-71.

45. Dum RP, Li C, Strick PL. Motor and nonmotor domains in the monkey dentate. Ann N Y Acad Sci. 2002;978:289-301.

46. Leiner HC, Leiner AL, Dow RS. The human cerebro-cerebellar system: its computing, cognitive and language skills. Behav Brain Res. 1991;44:113-28.

47. Marien P, Engelborghs S, Fabbro F, De Deyn PP. The lateralized linguistic cerebellum: a review and a new hypothesis. Brain Lang. 2001;79:580-600. 